\title{
Erratum to: Comparison of laparoscopic versus open left-sided hepatectomy for intrahepatic duct stones
}

Yong Chan Shin ${ }^{1}$ Jin-Young Jang ${ }^{1}$ Mee Joo Kang ${ }^{1}$ - Woohyun Jung ${ }^{1}$ • Jihoon Chang ${ }^{1} \cdot$ Ye Rim Chang ${ }^{1} \cdot$ Sun-Whe Kim ${ }^{1}$

Published online: 5 May 2015

(C) Springer Science+Business Media New York 2015

\section{Erratum to: Surg Endosc}

\section{DOI 10.1007/s00464-015-4200-3}

On page 5, right column, under Discussion, first paragraph, last sentence, the citation should be [17].

The online version of the original article can be found under doi:10. 1007/s00464-015-4200-3.

\section{Jin-Young Jang}

jangjy4@gmail.com

Yong Chan Shin

eggnut81@gmail.com

1 Department of Surgery, Seoul National University College of Medicine, 101 Daehak-ro, Chongno-gu, Seoul 110-744,

South Korea 